\title{
Real Space Imaging of Spin Stripe Domain Fluctuations in a Complex Oxide
}

\author{
Longlong Wu®, ${ }^{1,2, *}$ Yao Shen $\odot,{ }^{1}$ Andi M. Barbour, ${ }^{3}$ Wei Wang $\odot,{ }^{1}$ Dharmalingam Prabhakaran, ${ }^{4}$ Andrew T. Boothroyd, ${ }^{4}$ \\ Claudio Mazzoli, ${ }^{3}$ John M. Tranquada $\odot,{ }^{1}$ Mark P. M. Dean $\odot,{ }^{1}$ and Ian K. Robinson $\odot^{1,5, \dagger}$ \\ ${ }^{1}$ Condensed Matter Physics and Materials Science Department, Brookhaven National Laboratory, Upton, New York 11973, USA \\ ${ }^{2}$ Computational Science Initiative, Brookhaven National Laboratory, Upton, New York 11973, USA \\ ${ }^{3}$ National Synchrotron Light Source II, Brookhaven National Laboratory, Upton, New York 11973, USA \\ ${ }^{4}$ Department of Physics, University of Oxford, Clarendon Laboratory, Oxford OXI 3PU, United Kingdom \\ ${ }^{5}$ London Centre for Nanotechnology, University College London, London WC1E 6BT, United Kingdom
}

(Received 31 August 2021; accepted 19 November 2021; published 28 December 2021)

\begin{abstract}
Understanding the formation and dynamics of charge and spin-ordered states in low-dimensional transition metal oxide materials is crucial to understanding unconventional high-temperature superconductivity. $\mathrm{La}_{2-x} \mathrm{Sr}_{x} \mathrm{NiO}_{4+\delta}$ (LSNO) has attracted much attention due to its interesting spin dynamics. Recent $\mathrm{x}$-ray photon correlation spectroscopy studies have revealed slow dynamics of the spin order (SO) stripes in LSNO. Here, we applied resonant soft $\mathrm{x}$-ray ptychography to map the spatial distribution of the SO stripe domain inhomogeneity in real space. The reconstructed images show the SO domains are spatially anisotropic, in agreement with previous diffraction studies. For the SO stripe domains, it is found that the correlation lengths along different directions are strongly coupled in space. Surprisingly, fluctuations were observed in the real space amplitude signal, rather than the phase or position. We attribute the observed slow dynamics of the stripe domains in LSNO to thermal fluctuations of the SO domain boundaries.
\end{abstract}

DOI: 10.1103/PhysRevLett.127.275301

Strong correlations in transition metal oxides between charge, spin, orbital, and lattice degrees of freedom can lead to the emergence of symmetry-breaking novel quantum states, such as spin order (SO), charge order (CO), and superconductivity [1,2]. In these states, electrons exhibit collective behaviors, and their corresponding dynamics are often represented in the energy domain $[3,4]$. Understanding the driving forces behind the formation of SO and CO stripe states and their dynamics is crucial to understanding unconventional superconductivity [2]. However, due to the complex interplay between spin, charge, orbital, and lattice degrees of freedom, the microscopic mechanisms underlying these collective behaviors are mostly unknown and not captured well by theories based on effective single-electron interactions [5].

Stripe-ordered $\mathrm{La}_{2-x} \mathrm{Sr}_{x} \mathrm{NiO}_{4+\delta}$ (LSNO) received much attention due to its being a close analog of the high $T_{c}$ cuprate superconductors [1,6]. As a nonsuperconducting 214 nickelate family, LSNO presents an ideal model with complex interplay between spin-charge-lattice degrees of freedom, while exhibiting distinct spin and lattice ordering but only weak screening of low energy excitations [6-9]. Several prior studies have reported stripe transitions (i.e., $\mathrm{SO}$ or $\mathrm{CO}$ ) and related correlation lengths in LSNO $[7,8,10-16]$. The transition temperatures of these stripe orders have been determined by neutron studies to be $T_{\mathrm{SO}}=\sim 90, \sim 100$, and $\sim 120 \mathrm{~K}$ and $T_{\mathrm{CO}}=\sim 115, \sim 150$, and $\sim 190 \mathrm{~K}$ for compositions $x=0.20,0.225$, and 0.275 , respectively [17-19]. $T_{\mathrm{CO}}=\sim 150 \mathrm{~K}$ for $x=0.225$ was also confirmed with hard x-ray measurement [20]. Usually, the measured SO or CO stripes show limited spatial correlation, implying the presence of stripe domains. Based on a phenomenological Landau and cluster models, the spin and lattice coupling are predicted to play a fundamental role in stabilizing SO stripes [5,21-23]. Recent X-ray photon correlation spectroscopy (XPCS) measurements have observed slow fluctuations of SO stripes in particular LSNO crystals [24,25]. The time constant of the SO stripe fluctuations varies significantly with temperature and is slowest at $70 \mathrm{~K}$.

Here, we report the first resonant soft x-ray Bragg ptychography experiment to reveal the origin of the slow dynamics of spin order in a stripe-ordered $\mathrm{La}_{2-x} \mathrm{Sr}_{x} \mathrm{NiO}_{4+\delta}$ single crystal, with $x=0.225$ and $\delta=0.07$. Advances in coherent $\mathrm{x}$-ray imaging have led to our ability to make maps of these magnetic domain structures, which can be utilized to address the origin of these slow dynamical phenomena. X-ray ptychography is a powerful coherent imaging technique which can reconstruct complex amplitude and phase images with high spatial resolution and quantitative phase contrast from coherent diffraction patterns of the magnetic signal, measured on a raster grid of overlapping probe positions [26]. The SO domains of LSNO, which are phase shifted with respect to each other, have been successfully imaged in real space with a measurement time faster than the slow dynamics. This 


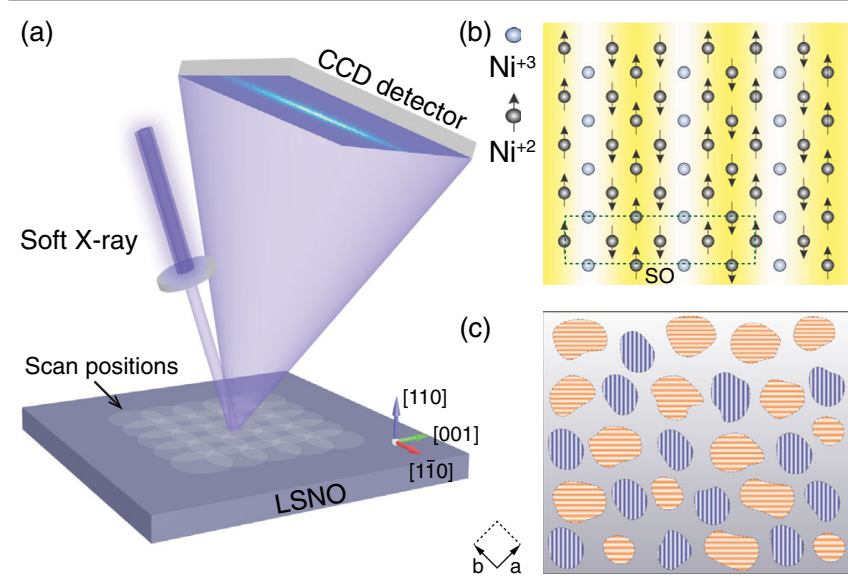

FIG. 1. (a) Schematic experimental soft x-ray Bragg ptychography experimental setup. (b) Cartoon of the spin stripe order in nickelate with simplified ionic doping level $x=0.25$. The doped holes reside on $\mathrm{Ni}$ and neighboring $\mathrm{O}$ ions, resulting in effective $\mathrm{Ni}^{3+}$ ions. The remaining $\mathrm{Ni}^{2+}$ ions are at high-spin state $(S=1)$. SO labels the unit cell of the spin superstructure and the shading shows the charge density distribution. (c) Real space cartoon of the formation of the mesostructured SO stripe domains with different modulation directions in the $\mathrm{NiO}_{2}$ plane.

allows us to discuss for the first time whether these fluctuations occur in the amplitude, phase, or relative positions of the domains.

The resonant soft $\mathrm{x}$-ray ptychography experiments were performed at the coherent soft x-ray 23-ID-1 beamline at National Synchrotron Light Source II. The energy of the incident x-ray beam was tuned to the Ni $L_{3}$ edge at $852 \mathrm{eV}$ with $\pi$ polarization, which greatly enhances the magnetic diffraction signal from SO (Supplemental Material [27]) and suppresses background. Before impinging on the LSNO sample, the monochromatic soft x-ray beam was shaped by an $8.0 \mu \mathrm{m}$ diameter pinhole, located $\sim 5.50 \mathrm{~mm}$ upstream, as shown in Fig. 1(a). During the measurements, the scattered $x$-ray signal was collected by a fast chargecoupled device detector $0.34 \mathrm{~m}$ away from the sample.

The LSNO single-crystal sample was grown by the floating-zone method with a Sr doping concentration of 0.225 [36]. After growth, the LSNO crystal was then polished so that its surface normal was closely along its [110] crystal direction to favor the measurements of the SO peak. Here, the reciprocal lattice units are defined as $\mathbf{Q}=(H, K, L)=(2 \pi / a, 2 \pi / b, 2 \pi / c)$, in the tetragonal unit cell with $I 4 / \mathrm{mmm}$ symmetry and the corresponding lattice constants $a=b=3.84 \AA$ and $c=12.65 \AA$. The Ni $L_{3}$ resonant Bragg reflection at wave vector $\mathbf{Q}=$ $(0.36,0.36,0)$ was used to probe SO. During the measurements, the temperature of the sample was set to $70 \mathrm{~K}$, where the SO stripe domains are more stable and correlated [24]. It is also well below the $\mathrm{SO}$ transition temperature, which is $\sim 100 \mathrm{~K}$. As shown in Fig. 1(a), the x-ray beam can generate coherent $\mathrm{x}$-ray diffraction patterns from $\mathrm{SO}$ close to symmetric backscattering, allowing a favorable reflection geometry for the raster-scan data acquisition of Bragg ptychography. The resonant coherent x-ray diffraction signal at the SO wave vector is very sensitive to magnetic scattering from the antiphase spin structure [Fig. 1(b)] and the modulation of the Ni valence electrons [37]. Figure S2 in the Supplemental Material [27] shows one representative coherent $\mathrm{x}$-ray diffraction pattern surrounding the $(0.36,0.36,0)$ SO Bragg peak, in which SO stripe domains interfere with each other, leading to a complex "speckle" pattern.

In nickelates, $\mathrm{SO}$ and $\mathrm{CO}$ coexist at low temperature, where doped charge carriers form one-dimensional stripes in concert with spin modulations with double the $\mathrm{CO}$ period, as shown in Fig. 1b [38-41]. The charge and spin stripes, which simultaneously break translational and rotational symmetry, are coupled and aligned along the "diagonal" direction, i.e., $45^{\circ}$ with respect to the $\mathrm{Ni}-\mathrm{O}$ bond direction [41]. Figure 1(c) schematically shows mesostructured "stripe" domains in an $\mathrm{NiO}_{2}$ plane associated with both the incommensurate $\mathrm{SO}$ wave vector $\mathbf{Q}_{H H}=(0.36,0.36)$ that we measure as well as $\mathbf{Q}_{H \bar{H}}=$ $(0.36,-0.36)$ that are not detected in this geometry.

The soft x-ray ptychography experiments used a wellpolished straight-walled pinhole fabricated by the focused ion beam method (Supplemental Material [27]). This avoids complications from the rough borders of the pinhole, prepared by laser ablation alone, which are found to introduce strong arcs and flares in the coherent diffraction [42]. The total scanning time for each $21 \times 21$ point grid ptychography scan was $\sim 23 \mathrm{~min}$, with an exposure time of $2 \mathrm{~s}$ per grid point, which is achieved by scanning the pinhole as shown in Fig. 1(a). The ptychographic reconstructions were computed using a GPU-accelerated iterative phase retrieval approach (Supplemental Material [27]). Because the soft $\mathrm{x}$ rays have a smaller penetration depth, $\sim 69 \mathrm{~nm}$ (Supplemental Material [27]) than the correlation length of SO domains, the diffraction pattern is effectively two dimensional; if the sample Bragg angle is changed the speckle pattern just shifts rather than evolves. Therefore, the Ewald cut through reciprocal space associated with the geometry of Fig. 1(a) fully samples the 2D cross section of the extended 3D Bragg peak distribution. So, despite the fact we are performing a "Bragg ptychography" experiment, we do not suffer difficulties of missing data [43] and can safely interpret the results as a 2D projection on the sample surface plane.

Figure 2(a) shows the reconstructed complex-valued wavefront of the illuminating beam aligned to the plane of the incident x-ray beam. The concentric fringes in the wavefront can be attributed to Fresnel propagation, and since the image is complex, it was numerically propagated back to the pinhole plane [44], shown in Fig. 2(b), which is indeed consistent with its scanning electron microscope (SEM) image [Fig. 2(c)]. Additionally, the horizontal cut 


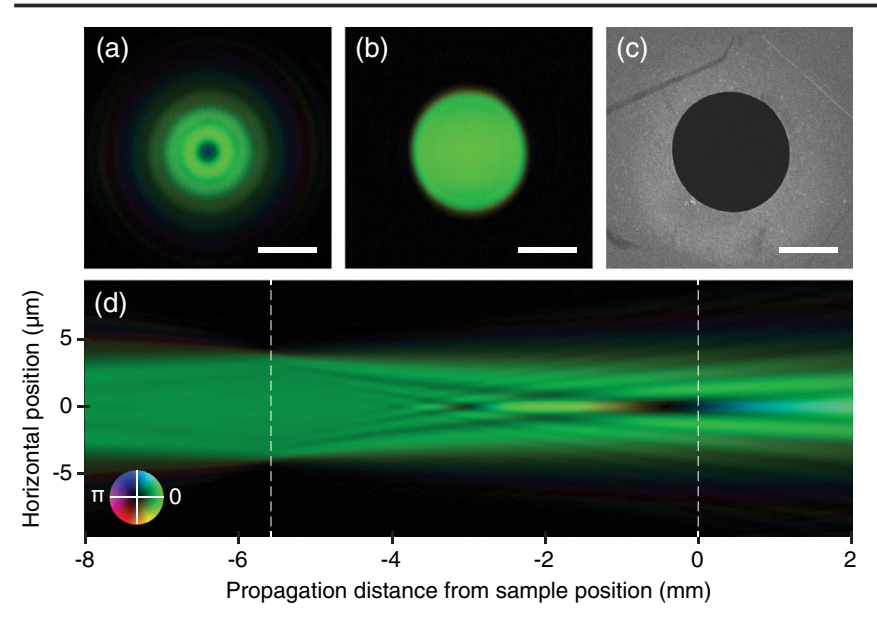

FIG. 2. (a) Reconstructed complex-valued wavefront of x-ray probe along the incident x-ray direction at the LNSO sample position. Here, the amplitude is mapped by the image brightness, and the corresponding phase is mapped by hue. (b) Computed free space propagation of the reconstructed soft x-ray probe to the plane where the pinhole was placed. (c) SEM image of the $8 \mu \mathrm{m}$ pinhole. (d) Horizontal cut through the propagated wavefield, displaying the waist of the soft $\mathrm{x}$-ray beam in the pinhole plane. Here, the left dashed lines show the position of the pinhole and the right dashed line shows the LSNO sample position. Scale bars are $4 \mu \mathrm{m}$.

through the complex caustic profile, given in Fig. 2(d), can be used to precisely deduce the distance between the LSNO sample and the pinhole, which is $5.57 \mathrm{~mm}$ [44].

Figure 3 shows the corresponding real space ptychographic images of the LSNO sample, measured at the SO peak and transformed into projections on the surface plane. A picture of close-packed elongated domains is clearly seen covering most of the field of view. The estimated resolution for these images is $\sim 23 \mathrm{~nm}$. The lengths and widths of the domains correspond roughly with the measured SO correlation lengths in the [110] and [001] directions [24]. The SO stripes along [110] will contribute to the image in our experiments, while the stripes along [110] will be dark. As one can see, each domain generally has a well-defined phase which reflects the relative position of its average stripes along the $\mathbf{Q}$ vector of the SO Bragg peak which is normal to the surface plane. A few domains show a sharp $\pi / 2$ phase-jump boundary, indicating coherence across a defect. The dark patches in Fig. 3 could be regions of (110) domains, but more likely represent surface damage due to crystal polishing, discussed below.

To elucidate the slow fluctuation of the SO domains, and to explore the general stability of the SO stripes, timedependent real space ptychographic images were made over $4 \mathrm{~h}$ both immediately and with an excursion to another part of the sample and returning. These are displayed in Fig. 3 as a function of indicated time at a fixed temperature (70 K). Because of the limited penetration depth of the soft $\mathrm{x}$ rays, the images shown in Fig. 3 are assumed to contain

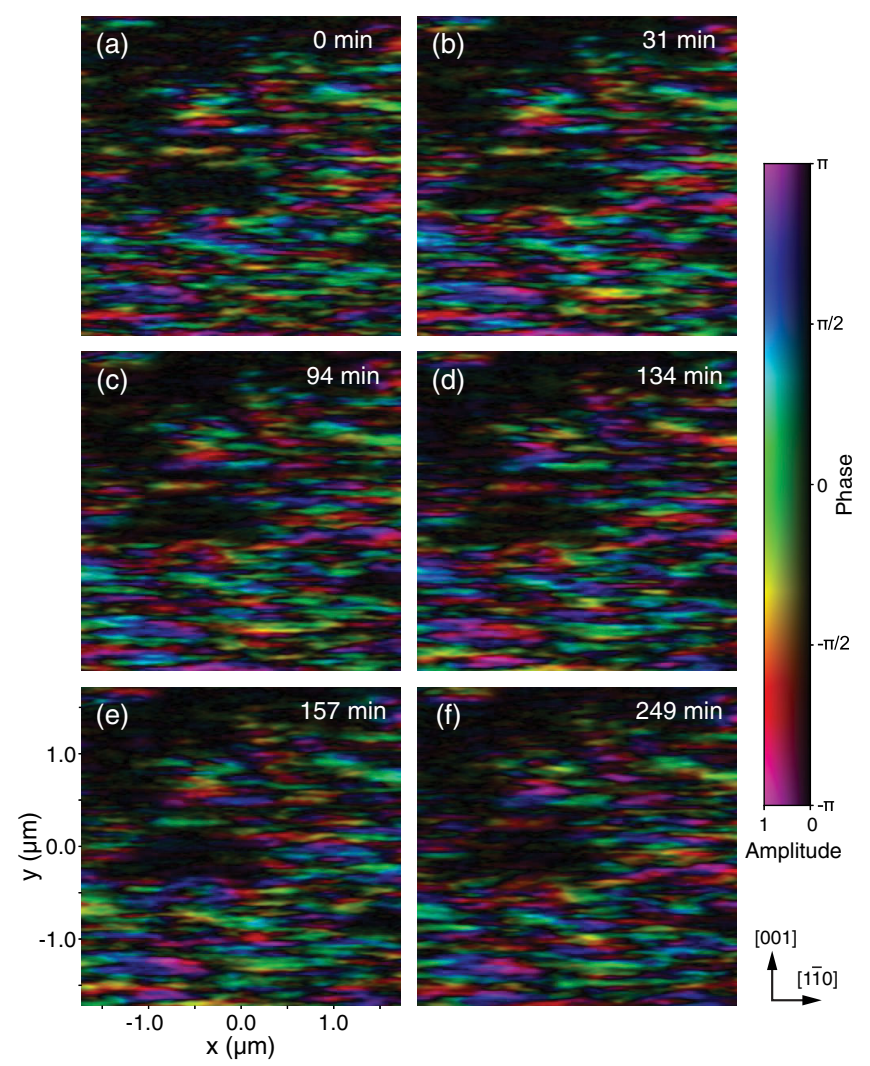

FIG. 3. (a)-(f) Bragg ptychography images of the reconstructed "stripe" domain structure in LSNO at $70 \mathrm{~K}$ at the indicated times during the experiment. The color scale is used to show the complex amplitude of the image, with the magnitude given by the brightness and the phase by the hue.

no more than one layer of SO stripe domains, which would be bright or dark. However, the estimated ratio of large areas of close packed (110) domains in Fig. 3 is 0.62 (Supplemental Material [27]), suggesting this is not true near the surface. Since the surface of a material always breaks inversion symmetry and results in inherent strain fields, it is possible the equal ratio of SO stripe domains along the two different directions is no longer kept.

The images in Fig. 3 appear locally similar, and the fluctuations are hard to see by direct examination. For fluctuating SO stripe domains, there are three different ways fluctuations might be considered to occur: in the amplitude, phase, or relative positions of the domains in the image. "Sliding" is a widely discussed excitation mechanism of charge or spin density waves $[45,46]$. This would manifest itself as changes of phase or domain position, corresponding to sliding perpendicular or parallel to the surface, respectively. In the time-resolved image in Fig. 3, no obvious trends with time were seen for the phases or the domain positions. Instead, and surprisingly, the amplitude was found to vary. This is shown in Fig. 4(a) as onedimensional amplitude profiles taken along [001] in each image at the same position. The profile of these lines can be seen to change as a function of time. We consider that this 

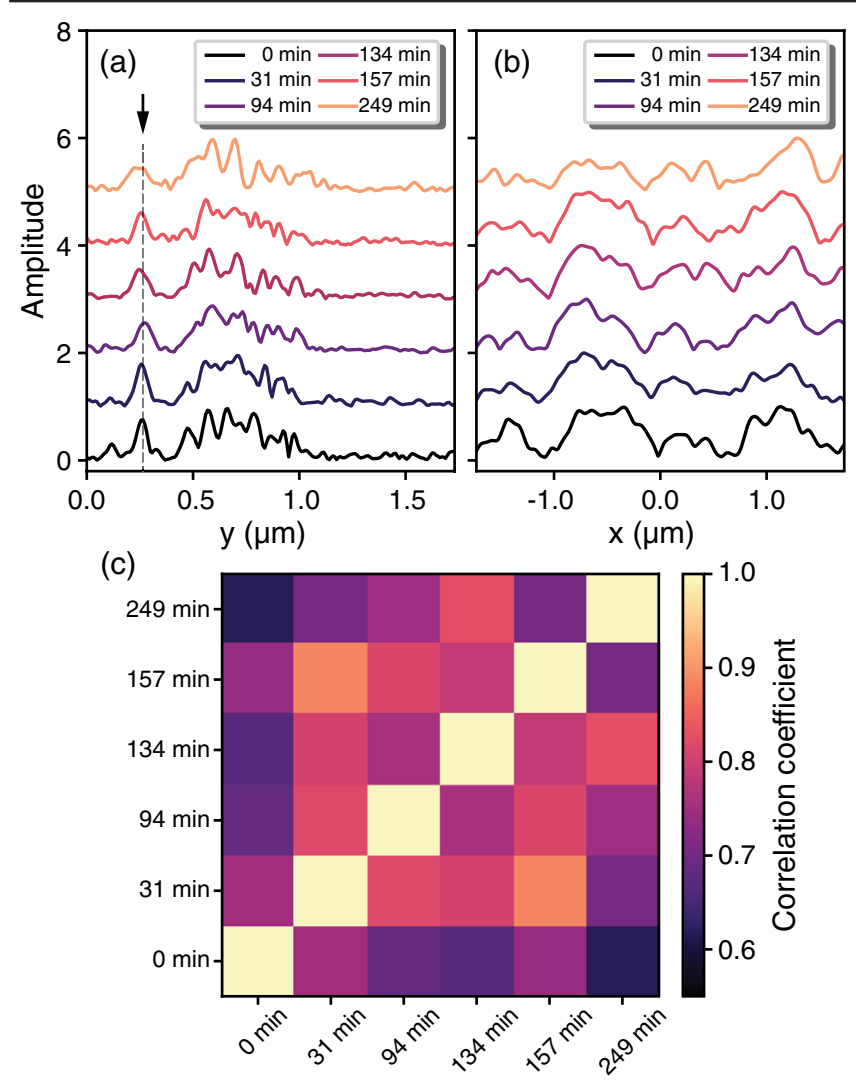

FIG. 4. (a) Extracted SO domain amplitude profiles along the vertical direction from the reconstructed real space images at $x=$ $-0.4 \mu \mathrm{m}$ from the reconstructed real space images in Fig. 3.

(b) Profiles along the horizontal direction at $y=0.35 \mu \mathrm{m}$.

(c) Cross-correlation plot of SO stripe amplitudes over the whole region of Fig. 3 at $70 \mathrm{~K}$.

results from changes in the three-dimensional volumes of the SO-ordered regions in the LSNO host lattice which undergo thermal fluctuation near the second-order SO phase transition. As the domain volume changes, the image amplitude would be expected to follow. This might be caused by individual spins flipping between adjacent domains. As indicated by the black arrow in Fig. 4(a), we also notice the center position changing of one isolated SO domain, which might be a rare example of the sliding effect, where the motion is $\sim 50 \mathrm{~nm}$. Sliding of SO domains along the normal [110] direction would also be detected as phase changes in the domain images of Fig. 3. Close inspection of Fig. 3 does indeed reveal a few domains which change phase, for example at $(x, y)$ position $(0.3,-1.2)$ which shows a $\sim 1$ radian change; this corresponds to a net motion of the SO domain by $\sim 0.35 \mathrm{~nm}$. However, sliding effects cannot account for the observed changes in the amplitude profiles of SO domains, which make the largest contribution to SO stripe domain fluctuation. Furthermore, Fig. 4(b) shows the corresponding one-dimensional lines are taken along [11̄0] with similar profile changes observed.

To further quantify the timescale of the SO domain fluctuations, Fig. 4(c) shows the calculated correlation coefficient (Supplemental Material [27]) as a heat map for all time pairs. The correlation drops off systematically with measurement time difference. This is consistent with our picture of size fluctuations of the SO domains, caused by individual spins flipping at the domain walls between them. Small changes in domain sizes will show up in the amplitude signal with finite image resolution. Just a few
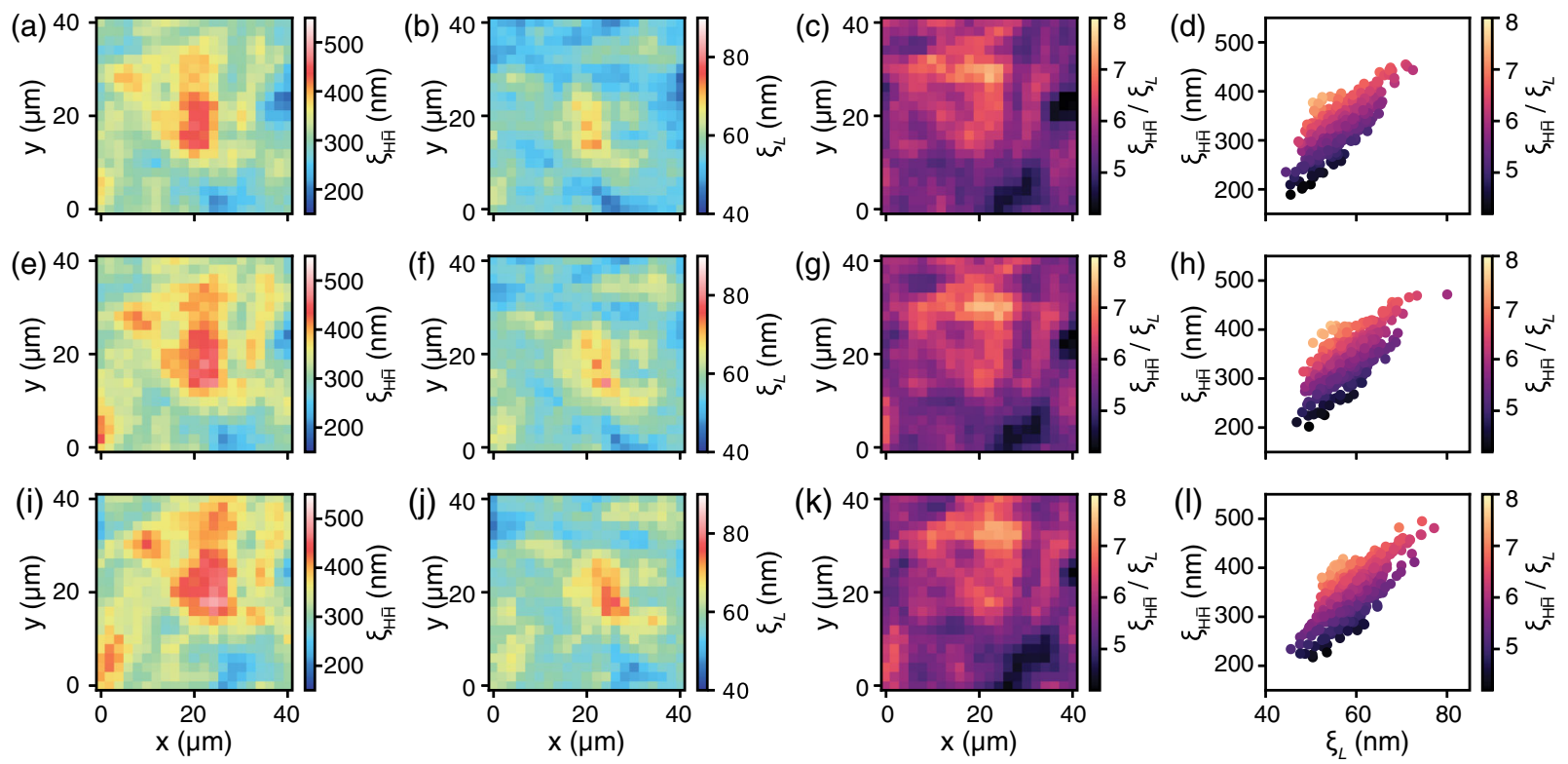

FIG. 5. (a),(e),(i) SO domain sizes extracted directly from the diffraction patterns along [110]. (b),(f),(j) Same along [001]. (c),(g),(k) The ratio between the two directions as a function of the scanning position. (d),(h),(l) The corresponding ratio distribution. (a)-(d), (e)-(h), and (i)-(l) were at 0,31 , and $94 \mathrm{~min}$, respectively. 
spins need to fluctuate for this change to occur, whereas an entire domain of spins must change to undergo sliding.

The SO stripe domain size can also be derived directly from the width of the coherent diffraction patterns. This allowed us to prepare spatial maps, shown in Fig. 5, of the domain sizes from the same data used for ptychography. The data of each ptychography frame were fitted by an anisotropic Lorentzian squared shape along the [110] and [001] directions (Supplemental Material [27]). The formal correlation length, defined as $\xi=2 \pi / \mathrm{FWHM}$, agrees well with the values found in Fig. 3 and so can be identified with the domain sizes in each direction. Figure 5 shows the SO domains size over a statistical region much larger than that in Fig. 3, and closer to that of the full ptychographic image in Fig. S4.

Figure 5 also shows distinct diagonal lines of low correlation length in the maps. These striking features are even more apparent in the high-resolution ptychography images of Fig. S4 where they appear as dark straight lines resembling the scratches due to crystal polishing. The disappearance of the SO domains in these regions may result from the surface damage of the sample due to polishing. It should also be mentioned that the appearance of these scratches in each ptychographic image allowed us to precisely align each of the six close-up images given in Fig. 3, for which the lateral positions suffered thermal drift. While it is possible the scratches are filled by (11)0) SO stripe domains, we suspect damage is more likely.

Figure 5 further confirms that the correlation length $\xi_{H \bar{H}}$ of SO along [1 $1 \overline{1} 0]$ is much larger than the correlation length $\xi_{L}$ of SO along [001], as also seen in the ptychographic reconstructed images. Figures 5(a) and 5(b) show that the SO domain sizes along both directions are inhomogeneously distributed. The coupling of spins within each $\mathrm{NiO}_{2}$ plane (in plane,) is expected to be much stronger than between spins in neighboring planes (out of plane, [001]) [47], as can be seen in the ratio of domain sizes along [1 $1 \overline{1} 0]$ and [001], shown in Fig. 5(c). The domain size ratio can be seen to vary as a function of the x-ray scanning position. Lastly, Fig. 5(d) shows a scatter plot of the ratio of the SO domain sizes, along the two correlation lengths. As one can see, the domain dimensions along the two directions are strongly correlated. Despite a significant growth in the SO domain size along both [1 $1 \overline{1} 0]$ and [001] with time, the trend in the ratio is preserved. Thus, though the coupling of spins within each $\mathrm{NiO}_{2}$ plane is much stronger than between neighboring planes, both couplings of spins affect the SO domains size.

Based on the results above, the SO fluctuation mechanisms are complicated. From both the ptychographic results in real space and peak fitting results in reciprocal space, we clearly show that these fluctuations are mainly coming from dynamics in the size (i.e., the amplitude) of the SO domains. This is important because it implies that the domain boundaries migrate by spin flipping during thermal fluctuation. Some sliding of SO domains could also be contributing to the fluctuation.

In summary, resonant soft x-ray ptychography has been applied to image spin-ordered (SO) stripes in a sample of composition $\mathrm{La}_{1.775} \mathrm{Sr}_{0.225} \mathrm{NiO}_{4.07}$ (LSNO), where real space domain images showing the size and spatial inhomogeneity of the domains are observed. Close to the surface, SO stripes show a preferred [110] orientation, which may be related to strain. In these real space ptychographic images, we find that the slow fluctuations are mainly coming from changes in the SO stripe's domain size, seen in the image amplitude signal because of limited resolution. At the level of our data, we see that just one of the three possible fluctuation channels is active and not the other two. The use of real space imaging allows the elucidation of further details of domain fluctuations first seen by XPCS. Furthermore, we find strong correlation of the SO domain size along the two observed directions. Our results open a new route to study the complex interplay between lattice and spin degrees of freedom in quantum materials at cryogenic temperatures.

The work at Brookhaven National Laboratory (BNL) was supported by the U.S. Department of Energy (DOE), Office of Basic Energy Sciences, under Contract No. DESC0012704. The work at UCL and Oxford University was supported by EPSRC. This research used the 23-ID-1 beamline of the National Synchrotron Light Source II, a U.S. DOE Office of Science User Facility operated for the DOE Office of Science by BNL under Contract No. DESC0012704.

*1wu@bnl.gov

†irobinson@bnl.gov

[1] D. F. Agterberg, J. C. S. Davis, S. D. Edkins, E. Fradkin, D. J. Van Harlingen, S. A. Kivelson, P. A. Lee, L. Radzihovsky, J. M. Tranquada, and Y.X. Wang, Annu. Rev. Condens. Matter Phys. 11, 231 (2020).

[2] R. Comin and A. Damascelli, Annu. Rev. Condens. Matter Phys. 7, 369 (2016).

[3] J. M. Tranquada, P. Wochner, and D. J. Buttrey, Phys. Rev. Lett. 79, 2133 (1997).

[4] G. Fabbris, D. Meyers, L. Xu, V. M. Katukuri, L. Hozoi, X. Liu, Z. Y. Chen, J. Okamoto, T. Schmitt, A. Uldry, B. Delley, G. D. Gu, D. Prabhakaran, A. T. Boothroyd, J. van den Brink, D. J. Huang, and M. P. M. Dean, Phys. Rev. Lett. 118, 156402 (2017).

[5] T. Hotta and E. Dagotto, Phys. Rev. Lett. 92, 227201 (2004).

[6] J. M. Tranquada, B. J. Sternlieb, J. D. Axe, Y. Nakamura, and S. Uchida, Nature (London) 375, 561 (1995).

[7] M. Filippi, B. Kundys, S. Agrestini, W. Prellier, H. Oyanagi, and N. L. Saini, J. Appl. Phys. 106, 104116 (2009).

[8] C. H. Chen, S. W. Cheong, and A.S. Cooper, Phys. Rev. Lett. 71, 2461 (1993). 
[9] S. M. Hayden, G. H. Lander, J. Zarestky, P. J. Brown, C. Stassis, P. Metcalf, and J. M. Honig, Phys. Rev. Lett. 68, 1061 (1992).

[10] S. W. Cheong, H. Y. Hwang, C. H. Chen, B. Batlogg, L. W. Rupp, Jr., and S. A. Carter, Phys. Rev. B 49, 7088 (1994).

[11] S. H. Lee and S. W. Cheong, Phys. Rev. Lett. 79, 2514 (1997).

[12] W. Bao, R. J. McQueeney, R. Heffner, J. L. Sarrao, P. Dai, and J. L. Zarestky, Phys. Rev. Lett. 84, 3978 (2000).

[13] M. E. Ghazi, P. D. Spencer, S. B. Wilkins, P. D. Hatton, D. Mannix, D. Prabhakaran, A. T. Boothroyd, and S. W. Cheong, Phys. Rev. B 70, 144507 (2004).

[14] R. Kajimoto, T. Kakeshita, H. Yoshizawa, T. Tanabe, T. Katsufuji, and Y. Tokura, Phys. Rev. B 64, 144432 (2001).

[15] P. G. Freeman, A. T. Boothroyd, D. Prabhakaran, M. Enderle, and C. Niedermayer, Phys. Rev. B 70, 024413 (2004).

[16] M. Raczkowski, R. Fresard, and A. M. Oles, Phys. Rev. B 73, 094429 (2006).

[17] V. V. Sachan, D. J. Buttrey, J. M. Tranquada, J. E. Lorenzo, and G. Shirane, Phys. Rev. B 51, 12742 (1995).

[18] J. M. Tranquada, D. J. Buttrey, and V. V. Sachan, Phys. Rev. B 54, 12318 (1996).

[19] S. H. Lee, J. M. Tranquada, K. Yamada, D. J. Buttrey, Q. Li, and S. W. Cheong, Phys. Rev. Lett. 88, 126401 (2002).

[20] A. Vigliante, M. von Zimmermann, J. R. Schneider, T. Frello, N. H. Andersen, J. Madsen, D. J. Buttrey, D. Gibbs, and J. M. Tranquada, Phys. Rev. B 56, 8248 (1997).

[21] J. Zaanen and P. B. Littlewood, Phys. Rev. B 50, 7222 (1994).

[22] S. H. Lee, Y. C. Lai, C. H. Du, A. F. Siegenfeld, Y. J. Kao, P. D. Hatton, D. Prabhakaran, Y. X. Su, and D. J. Huang, Phys. Rev. B 92, 205114 (2015).

[23] S. Yamamoto, T. Fujiwara, and Y. Hatsugai, Phys. Rev. B 76, 165114 (2007).

[24] Y. Shen, G. Fabbris, H. Miao, Y. Cao, D. Meyers, D. G. Mazzone, T. A. Assefa, X. M. Chen, K. Kisslinger, D. Prabhakaran, A. T. Boothroyd, J. M. Tranquada, W. Hu, A. M. Barbour, S. B. Wilkins, C. Mazzoli, I. K. Robinson, and M. P. M. Dean, Phys. Rev. Lett. 126, 177601 (2021).

[25] A. Ricci, N. Poccia, G. Campi, S. Mishra, L. Muller, B. Joseph, B. Shi, A. Zozulya, M. Buchholz, C. Trabant, J. C. T. Lee, J. Viefhaus, J. B. Goedkoop, A. A. Nugroho, M. Braden, S. Roy, M. Sprung, and C. Schussler-Langeheine, Phys. Rev. Lett. 127, 057001 (2021).

[26] F. Pfeiffer, Nat. Photonics 12, 9 (2018).

[27] See Supplemental Material at http://link.aps.org/ supplemental/10.1103/PhysRevLett.127.275301 for detailed information about sample preparation, pinhole fabrication, soft x-ray measurements, penetration depth estimation, ptychography reconstruction, SO domain over a large scale, filling fraction of SO domain estimation, and correlation coefficient, which includes Refs. [28-35].

[28] M. P. Dean, G. Dellea, R. S. Springell, F. Yakhou-Harris, K. Kummer, N. B. Brookes, X. Liu, Y. J. Sun, J. Strle, T. Schmitt, L. Braicovich, G. Ghiringhelli, I. Bozovic, and J. P. Hill, Nat. Mater. 12, 1019 (2013).

[29] J. Daillant and A. Gibaud, X-Ray and Neutron Reflectivity, Lecture Notes in Physics (Springer, Berlin Heidelberg, 2009).

[30] B. L. Henke, E. M. Gullikson, and J. C. Davis, At. Data Nucl. Data Tables 54, 181 (1993).

[31] N. Burdet, X. Shi, J. N. Clark, X. Huang, R. Harder, and I. Robinson, Phys. Rev. B 96, 014109 (2017).

[32] A. Maiden, D. Johnson, and P. Li, Optica 4, 736 (2017).

[33] R. W. Gerchberg and W. O. Saxton, Optik (Stuttgart) 35, 237 (1972).

[34] P. Thibault, M. Dierolf, A. Menzel, O. Bunk, C. David, and F. Pfeiffer, Science 321, 379 (2008).

[35] A. M. Maiden, M. J. Humphry, M. C. Sarahan, B. Kraus, and J.M. Rodenburg, Ultramicroscopy 120, 64 (2012).

[36] D. Prabhakaran, P. Isla, and A. T. Boothroyd, J. Cryst. Growth 237-239, 815 (2002).

[37] C. Schussler-Langeheine, J. Schlappa, A. Tanaka, Z. Hu, C. F. Chang, E. Schierle, M. Benomar, H. Ott, E. Weschke, G. Kaindl, O. Friedt, G. A. Sawatzky, H. J. Lin, C. T. Chen, M. Braden, and L. H. Tjeng, Phys. Rev. Lett. 95, 156402 (2005).

[38] P. Wochner, J. M. Tranquada, D. J. Buttrey, and V. Sachan, Phys. Rev. B 57, 1066 (1998).

[39] J. M. Tranquada, J. E. Lorenzo, D. J. Buttrey, and V. V. Sachan, Phys. Rev. B 52, 3581 (1995).

[40] M. Hücker, M. v. Zimmermann, R. Klingeler, S. Kiele, J. Geck, S. N. Bakehe, J. Z. Zhang, J. P. Hill, A. Revcolevschi, D. J. Buttrey, B. Büchner, and J. M. Tranquada, Phys. Rev. B 74, 085112 (2006).

[41] A. M. Merritt, D. Reznik, V. O. Garlea, G. D. Gu, and J. M. Tranquada, Phys. Rev. B 100, 195122 (2019).

[42] M. Dierolf, P. Thibault, A. Menzel, C. M. Kewish, K. Jefimovs, I. Schlichting, K. Von Konig, O. Bunk, and F. Pfeiffer, New J. Phys. 12, 035017 (2010).

[43] S. O. Hruszkewycz, M. Allain, M. V. Holt, C. E. Murray, J. R. Holt, P. H. Fuoss, and V. Chamard, Nat. Mater. 16, 244 (2017).

[44] P. Thibault, M. Dierolf, O. Bunk, A. Menzel, and F. Pfeiffer, Ultramicroscopy 109, 338 (2009).

[45] I. I. Tutto and A. Zawadowski, Phys. Rev. Lett. 60, 1442 (1988).

[46] V. L. Jacques, C. Laulhe, N. Moisan, S. Ravy, and D. Le Bolloc'h, Phys. Rev. Lett. 117, 156401 (2016).

[47] A. Maity, R. Dutta, and W. Paulus, Phys. Rev. Lett. 124, 147202 (2020). 